\title{
Market Share Instability In The U.S. Airline Industry
}

Philippe Barla, (philippe.barla@ecn.ulaval.ca), Université Laval

\begin{abstract}
In this paper, we use Market Share Instability (MSI) as a measure of the intensity of competition among airlines on a route. Using a panel of 400 U.S. domestic routes over the 1987-1993 period, we try to explain variations in MSI. We find that MSI is higher in moderately concentrated routes and in routes that include a bankrupt carrier. Airport dominance increases MSI if the dominant airline faces small competitors. Finally, we show that MSI was lower in the early nineties than in the late eighties.
\end{abstract}

